\title{
Influência da redução da força muscular em idosos nos parâmetros biomecânicos da marcha
}

\author{
Deborah Hebling SPINOSO* \\ Camilla Zamfollini HALLAL ** \\ Nise Ribeiro MARQUES* \\ Aline Harumi KARUKA ${ }^{* * *}$ \\ Fernanda Cristina MILANEZ ${ }^{* * * * *}$ \\ Mauro GONÇALVES ${ }^{* * * *}$
}

\author{
'Departamento \\ de Fisioterapia e \\ Terapia Ocupacional, \\ Universidade Estadual \\ Paulista, Marília, SP, \\ Brasil. \\ "Departamento \\ de Fisioterapia, \\ Universidade Federal \\ de Uberlândia, \\ Uberlândia, MG, Brasil. \\ "Departamento \\ de Fisioterapia, \\ Universidade Estadual \\ Paulista, Presidente \\ Prudente, SP, Brasil. \\ "'Departamento de \\ Educação Física, \\ Universidade Estadual \\ Paulista, Rio Claro, SP, \\ Brasil.
}

\section{Resumo}

0 objetivo do estudo foi analisar como a diminuição da força muscular dos extensores de joelho afeta 0 comportamento de variáveis biomecânicas durante a marcha simples e com dupla tarefa. Participaram do estudo 15 jovens (GJ), 14 idosas com força normal (GIN) e 13 idosas com força reduzida (GIR). 0 nível de força foi determinado pelo torque extensor de joelho, considerando o limiar de $1.5 \mathrm{NKg}^{-1}$. A avaliação biomecânica da marcha foi feita utilizando um sistema de análise 3D com uma plataforma de força. As condições analisadas foram marcha em velocidade de preferência (I), marcha em velocidade de preferência com carga (II), marcha em velocidade de preferência falando ao telefone (III), marcha em velocidade de preferência com obstrução da visão dos pés (IV) e marcha em velocidade de preferência máxima (V). Os resultados mostraram que GIR e GIN apresentaram alterações cinéticas e cinemáticas durante as condições de marcha com dupla tarefa motora e cognitiva em relação ao GJ. Entretanto apenas as variáveis taxa de propulsão e força de reação do solo vertical $(p<0.05)$ durante as condições de marcha com dupla tarefa foram capazes de diferenciar os grupos de idosos. Dessa maneira podemos concluir que a avaliação da marcha com dupla tarefa é capaz de diferenciar idosos com comprometimento de força muscular. Além disso, a força dos extensores de joelho é determinante dos parâmetros velocidade de marcha e força de reação do solo, sendo assim a fraqueza desse grupo muscular associado às alterações cinemáticas da marcha pode comprometer a execução de um padrão de marcha eficiente e seguro, tanto em condições simples como durante dupla-tarefa, resultando em maior risco de quedas e incapacidade funcional.

Palavras-Chave: Dupla-tarefa; Força de Reação do Solo; Cinemática.

\section{Introdução}

A habilidade de andar é uma tarefa complexa e exige uma perfeita harmonia do sistema sensorial, motor e cognitivo para produzir um padrão de marcha estável, eficiente e seguro ${ }^{1}$. Embora seja o movimento humano básico, é uma tarefa que exige atenção ${ }^{2}$.
Durante a maioria das atividades de vida diária, a marcha é realizada simultaneamente com outra tarefa motora ou cognitiva ${ }^{3}$. Essa sobreposição de tarefas, como por exemplo, quando a caminhada é acompanhada de superaçáo de obstáculos, uso de 
celular, resposta a mudança de cor nos semáforos, é denominada como dupla-tarefa ${ }^{2}$.

A dificuldade na realização dessas atividades com o envelhecimento está relacionada com diminuição da habilidade do sistema nervoso central em dividir a atenção para realizar duas tarefas concomitantemente de maneira eficiente, podendo resultar em maior risco de quedas nessa populaçãa ${ }^{4,5}$.

Todos os anos, cerca de 30 a 40\% dos indivíduos com mais de 60 anos de idade, vai cair, pelo menos uma vez e $50 \%$ dessas quedas ocorrem durante a marcha ${ }^{6}$. Os principais fatores de risco para quedas são a diminuição do equilíbrio, mobilidade e alterações nas variáveis cinéticas e cinemáticas de marcha ${ }^{7-9}$. Apesar de serem descritas na literatura, muitas vezes essas mudanças nas variáveis espaço temporais da marcha são apresentadas sem uma relação causal.

Entre as possíveis razóes para as modificações encontradas na marcha de idosos, a redução da força muscular tem sido considerada um dos principais fatores que pode ocasionar limitações funcionais durante a realização de tarefas diárias ${ }^{10-12}$.

Geralmente, indivíduos jovens possuem força de membro inferior suficiente para caminhar em velocidades moderadas e rápidas, ajustando o padrão de marcha de acordo com a necessidade da tarefa, sem prejudicar a eficiência do movimento ${ }^{13}$. Entretanto, a partir da meia idade os níveis de força

\section{Método}

\section{Sujeitos}

Foram recrutadas 42 voluntárias, divididas em dois grupos etários: jovens e idosas (TABELA 1). O grupo de jovens (GJ) foi composto por 15 voluntárias com idade entre 18-25 anos, estudantes universitárias, fisicamente ativas. O grupo de idosas foi composto por mulheres com idade entre 60-80 anos, participantes de grupo de atividade física para a comunidade e estas foram subdivididas em grupo de idosas com força muscular normal declinam progressivamente chegando a $15 \%$ na sexta década de vida e posteriormente atingindo uma perda de $30 \%$ por década até que esses indivíduos não tenham mais força suficiente para impulsionar o corpo para frente em uma velocidade funcional ${ }^{13,14}$. Assim, tem sido proposto o limiar de torque extensor de joelho de $1.5 \mathrm{Nm} \mathrm{Kg}^{-1}$ para identificar aqueles que tem risco de limitação de mobilidade e quedas, devido aos baixos níveis de força ${ }^{15-17}$.

Dessa maneira, a realização de investigaçóes sobre a relação da influência dos níveis de força muscular no padrão biomecânico de marcha dos idosos pode auxiliar a caracterizar melhor quais são as estratégias compensatórias adotadas por essa população durante a caminhada e assim proporcionar maior direcionamento para reabilitação desses indivíduos afim de melhorar o nível de independência e prevenção de incapacidades. O objetivo do estudo foi analisar como os níveis de força muscular afetam as variáveis cinéticas e cinemáticas durante condição de marcha simples e com dupla tarefa. A hipótese desse estudo é que idosos apresentarão modificaçóes tanto em parâmetros cinéticos e cinemáticos da marcha em relação aos jovens, e que essas alteraçóes serão mais acentuadas em idosos com baixa força muscular. Além disso, espera-se que em condiçóes de dupla-tarefa essas alteraçôes sejam mais evidenciadas.

TABELA 1 - Características dos sujeitos

\begin{tabular}{lccc}
\hline & GJ $(\mathbf{n}=\mathbf{1 5})$ & GIN $(\mathbf{n}=\mathbf{1 4})$ & GIR $(\mathbf{n}=\mathbf{1 3})$ \\
\hline Idade (anos) & $23.63 \pm 1.6$ & $69.13 \pm 5.4^{*}$ & $66.69 \pm 4.3^{\dagger}$ \\
Massa (kg) & $59.11 \pm 8.1$ & $65.00 \pm 6.8$ & $70.60 \pm 10.9^{\dagger}$ \\
\hline
\end{tabular}

(GIN) e grupo de idosas com força muscular reduzida (GIR), conforme descrito no item abaixo. Os critérios de elegibilidade desse estudo foram gênero feminino, não apresentar dor, fratura ou lesão grave em músculos e ligamentos nos seis meses pregressos ao estudo, bem como histórico de alteraçôes neurológicas, cardiovasculares ou respiratórias e ser capaz de caminhar de maneira independente. Todas as voluntárias assinaram o termo de consentimento e o estudo foi aprovado pelo Comitê de Ética local. 
Continuação

TABELA 1 - Características dos sujeitos

\begin{tabular}{lccc}
\hline & GJ $(\mathbf{n}=\mathbf{1 5})$ & GIN $(\mathbf{n}=\mathbf{1 4})$ & GIR $(\mathbf{n}=\mathbf{1 3})$ \\
\hline Estatura $(\mathrm{m})$ & $1.61 \pm 0.04$ & $1.54 \pm 0.05^{*}$ & $1.52 \pm 0.05^{\dagger}$ \\
Índice de Massa Corporal $\left(\mathrm{kg} \mathrm{m}^{-2}\right)$ & $22.44 \pm 2.2$ & $27.65 \pm 3.6^{*}$ & $30.18 \pm 4.2^{\dagger}$ \\
Torque extensor de joelho $\left(\mathrm{Nm} \mathrm{kg}^{-1}\right)$ & $2.45 \pm 0.52^{* \dagger}$ & $1.92 \pm 0.2$ & $1.24 \pm 0.2^{\S}$ \\
Número de quedas (por ano) & - & 0.28 & 1.6 \\
\hline
\end{tabular}

Valores de média \pm DP; "Diferença significativa entre GJ e GIN;

†Diferença significativa entre GJ e GIR;

§Diferença significativa entre GIR e GIN, $p>0.05$

\section{Procedimentos}

As participantes realizaram duas visitas ao laboratório, com intervalo de 2-7 dias entre eles ${ }^{18}$. Durante a primeira visita foi realizada a coleta de dados antropométricos, histórico de quedas nos últimos 12 meses e avaliaçáo do torque isométrico do membro inferior dominante (item 2.3) e familiarização com o teste de marcha. $\mathrm{O}$ limiar de torque isométrico extensor de joelho de $1.5 \mathrm{Nm} \mathrm{Kg}^{-1}$ foi utilizado para classificar as voluntárias idosas quanto ao nível de força muscular ${ }^{15}$. Quatorze voluntárias idosas apresentaram valores inferiores a $1,5 \mathrm{Nm} \mathrm{kg}^{-1}$ e foram classificadas para o grupo força muscular reduzida e treze mulheres apresentaram valores superiores a $1,5 \mathrm{Nm} \mathrm{kg}^{-1}$ sendo classificadas para o grupo força muscular normal. Durante a segunda visita ao laboratório, as voluntárias realizaram o teste de marcha (item 2.4) em diferentes condiçóes que simulavam atividades que fazem parte do cotidiano.

\section{Avaliaçáo do torque extensor de joelho}

A mensuração do torque isométrico extensor do joelho foi realizada utilizando o dinamômetro isocinético Biodex System 4 PRO (Biodex ${ }^{\circledR}$ ). As voluntárias foram posicionadas com o quadril em $90^{\circ}$ de flexão e joelho com $60^{\circ}$ de flexão $\left(0^{\circ}\right.$ extensão completa), com o eixo articular do dinamômetro alinhado com o eixo articular do joelho. A alavanca do dinamômetro foi posicionada acima dos maléolos lateral/medial do membro avaliado. O tronco e o membro inferior contralateral foram fixados por cintos.

Duas contrações isométricas máximas foram realizadas por um período de cinco segundos, com intervalo de 30 segundos entre elas ${ }^{19}$. O intervalo entre cada série de contraçóes foi de dois minutos. Encorajamento verbal padronizado e feedback visual foram fornecidos às voluntárias durante a avaliação do torque isométrico.

\section{Avaliaçáo da marcha}

A avaliação da marcha foi realizada utilizando uma plataforma de força OR6-6 (AMTI $\left.{ }^{\circledast}, 100 \mathrm{~Hz}\right)$ em uma passarela de 14 metros de comprimento e 2 metros de largura, sendo que os dois iniciais e finais da passarela foram desconsiderados para evitar possíveis influências do processo de aceleração e desaceleração da marcha. Para determinação das variáveis temporais da marcha foram utilizados dois sensores de pressão FootSwitch (Noraxon ${ }^{\circledR}$ ) posicionados na base do hálux e calcâneo. Para a determinação das variáveis espaciais, foram utilizados marcadores fotorreflexivos posicionados bilateralmente no tendão do calcâneo e maléolos.

O protocolo de avaliação foi composto de cinco diferentes condiçóes de marcha que simulavam situaçóes que fazem parte do cotidiano, sendo elas: condição I: as voluntárias foram orientadas a caminhar na passarela na mesma velocidade que realizam suas atividades de vida diária; condição II: as voluntárias foram orientadas a caminhar na passarela enquanto carregavam cargas correspondentes $10 \%$ do peso corporal em sacolas similares às de compras, divididas em ambos os membros superiores; condição III: as voluntárias foram orientadas a caminhar na passarela enquanto respondiam ao celular questóes simples relacionadas às suas atividades de vida diárias feitas por um examinador; condição IV: as voluntárias foram orientadas a caminhar na passarela carregando uma placa opaca com $29,5 \mathrm{~cm}$ de comprimento e $5 \mathrm{~cm}$ de altura; e condição V: as voluntárias foram orientadas a caminhar o mais rápido possível sem correr e sem a sensação que iriam tropeçar ou cair.

As participantes realizaram dez tentativas para cada condição de marcha proposta.

\section{Análise das variáveis espaciais e temporais da marcha}

Para análise das variáveis cinemáticas da marcha foram utilizadas 6 passadas consecutivas 
de cada condição de marcha ${ }^{20}$. Os parâmetros espaciais da marcha foram calculados por meio do processamento dos dados cinemáticos obtidos pelo software Nexus (Vicon ${ }^{\circledR}$ ). O comprimento do passo foi determinado por meio da distância, no eixo antero-posterior, entre os marcadores fotorreflexivos do calcâneo direito e esquerdo. A largura do passo foi calculada pela distância, no eixo médio-lateral, entre os marcadores fotorreflexivos posicionados nos maléolos direito e esquerdo. Para análise dos parâmetros temporais da marcha, o tempo de apoio foi determinado pelo tempo decorrido entre o contato inicial do calcâneo com o solo até a retirada do hálux. O tempo de balanço foi calculado pelo tempo entre a retirada do hálux do solo até esse mesmo pé tocar o solo novamente. O tempo de passada foi determinado pelo tempo entre o toque do calcâneo com o solo até o próximo toque de calcâneo do mesmo membro (FIGURA 1). A velocidade da marcha foi calculada por meio da divisão entre a distância e o tempo gasto para percorrer a passarela.

Fase de Apoio

Passada

Fase de Balanço

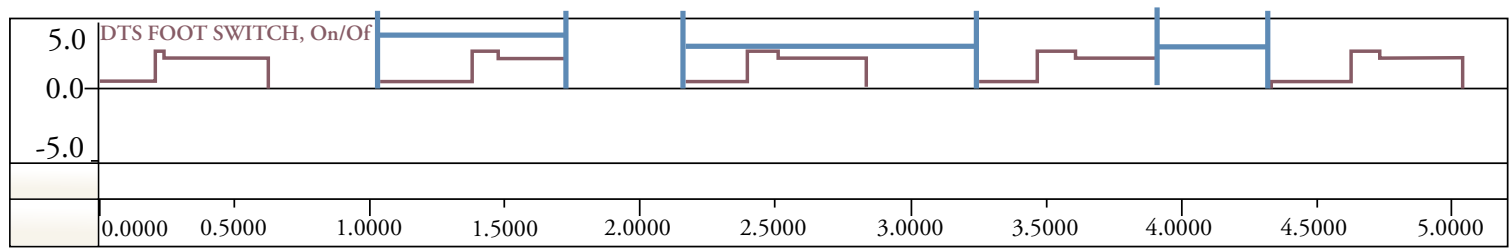

FIGURA 1 - Sinal dos sensores de pressão FootSwitch para determinação das variáveis temporais da marcha.

TABELA 2 - Comparação das variáveis cinéticas entre jovens, idosas com força normal e idosas com força reduzida em diferentes condições de marcha.

Valores de média \pm desvio padrão. *diferença significativa em relação ao grupo jovem. §diferença significativa entre idosos com força normal e força reduzida ${ }^{\dagger}$ diferença significativa em relação a condição de marcha normal. GJ = grupo jovens. $\mathrm{GIN}=$ grupo idosos força normal. $\mathrm{GIR}=$ grupo idosos força reduzida, $p>0.05$

\begin{tabular}{|c|c|c|c|c|c|}
\hline & & \multicolumn{4}{|c|}{ Variáveis cinéticas da marcha } \\
\hline & & $\begin{array}{l}\text { PFRSV aceitaçáo } \\
\text { do peso }\left(\mathrm{Nm} \mathrm{kg}^{-1}\right)\end{array}$ & $\begin{array}{l}\text { PFRSV propulsáo } \\
\left(\mathrm{Nm} \mathrm{kg}{ }^{-1}\right)\end{array}$ & $\begin{array}{c}\text { Taxa aceitaçáo do peso } \\
\left.(\mathrm{Nm} \mathrm{kg} \mathrm{s})^{-1}\right)\end{array}$ & $\begin{array}{l}\text { Taxa de propulsáo } \\
\qquad\left(\mathrm{Nm} \mathrm{kg} \mathrm{s}^{-1}\right)\end{array}$ \\
\hline \multicolumn{6}{|c|}{ Condiçóes de Marcha } \\
\hline \multirow[t]{3}{*}{ Normal } & GJ & $1.07 \pm 0.08$ & $1.32 \pm 0.15$ & $7.70 \pm 1.74$ & $5.27 \pm 1.41$ \\
\hline & GIN & $1.04 \pm 0.08$ & $1.22 \pm 0.16$ & $6.43 \pm 1.78$ & $4.22 \pm 1.82$ \\
\hline & GIR & $0.99 \pm 0.12$ & $1.14 \pm 0.18$ & $6.29 \pm 1.04^{*}$ & $4.33 \pm 1.22$ \\
\hline \multirow[t]{3}{*}{ Carga } & GJ & $1.15 \pm 0.09$ & $1.38 \pm 0.11$ & $8.49 \pm 2.90$ & $6.01 \pm 1.38$ \\
\hline & GIN & $1.09 \pm 0.09$ & $1.26 \pm 0.17$ & $6.73 \pm 1.97$ & $5.62 \pm 2.05$ \\
\hline & GIR & $1.12 \pm 0.04$ & $1.16 \pm 0.16$ & $7.18 \pm 1.65$ & $4.51 \pm 1.47^{*}$ \\
\hline \multirow[t]{3}{*}{ Celular } & GJ & $1.08 \pm 0.06$ & $1.36 \pm 0.10$ & $8.14 \pm 1.92$ & $5.32 \pm 1.29$ \\
\hline & GIN & $1.02 \pm 0.05$ & $1.09 \pm 0.23$ & $4.78 \pm 1.85$ & $2.99 \pm 1.04$ \\
\hline & GIR & $1.02 \pm 0.04^{*}$ & $1.02 \pm 0.25^{\star \S}$ & $5.94 \pm 2.78^{*}$ & $3.40 \pm 1.74^{* \S}$ \\
\hline \multirow[t]{3}{*}{ Placa } & GJ & $1.11 \pm 0.06$ & $1.40 \pm 0.12$ & $7.37 \pm 2.04$ & $5.99 \pm 1.30$ \\
\hline & GIN & $1.03 \pm 0.12$ & $1.25 \pm 0.17$ & $6.44 \pm 1.87$ & $4.64 \pm 1.88$ \\
\hline & GIR & $1.07 \pm 0.11$ & $1.17 \pm 0.16^{\star \S}$ & $6.31 \pm 2.08$ & $4.29 \pm 1.13^{*}$ \\
\hline \multirow[t]{3}{*}{ Rápida } & GJ & $1.36 \pm 0.11$ & $1.98 \pm 0.20$ & $12.18 \pm 2.87$ & $9.28 \pm 2.06$ \\
\hline & GIN & $1.19 \pm 0.16^{*}$ & $1.54 \pm 0.15^{*}$ & $8.35 \pm 3.84^{*}$ & $6.03 \pm 1.62^{*}$ \\
\hline & GIR & $1.16 \pm 0.17^{*}$ & $1.48 \pm 0.15^{*}$ & $8.64 \pm 2.84^{*}$ & $4.92 \pm 1.65^{*}$ \\
\hline
\end{tabular}




\section{Análise das variáveis cinéticas da marcha}

Para a análise cinética da marcha foram consideradas as tentativas na qual toda a região plantar estava em contato com a plataforma de força. O componente vertical da força de reação do solo foi selecionado para analise pois tem se mostrado altamente reprodutível e confiável por apresentar uma forma bimodal caracterizada por dois picos máximos, que representam momentos importantes da fase de apoio da marcha ${ }^{21}$. O primeiro pico ocorre durante a fase de aceitação do peso e representa o contato do calcanhar com o solo; o segundo pico, por sua vez, se refere a fase de propulsão da marcha, responsável pela execuçáo do próximo passo ${ }^{22}$. Nesse sentido, para análise dos dados a fase de apoio da marcha foi dividida em fase de aceitação do peso que corresponde à primeira metade do período de contado do pé com o solo; e a fase de propulsão, que corresponde à segunda metade do período de contato do pé com o solo, conforme proposto por Stacoff et al. ${ }^{23}$ e LaRoche et al. ${ }^{20}$ (FIGURA 2). O pico de força de reação do solo vertical (PFRSV) foi determinado durante a fase de aceitação do peso e propulsão. A análise da taxa de aceitação do peso foi calculada por meio da inclinação da curva força versus tempo entre $10 \%$ a $90 \%$ do pico de força nessa fase; e a taxa de propulsão foi determinada pela inclinação da curva força versus tempo entre $90 \%$ a $10 \%$ do pico de força nessa fase ${ }^{19,21}$. A normalização dos dados foi obtida em relaçáo ao peso corporal das voluntárias.

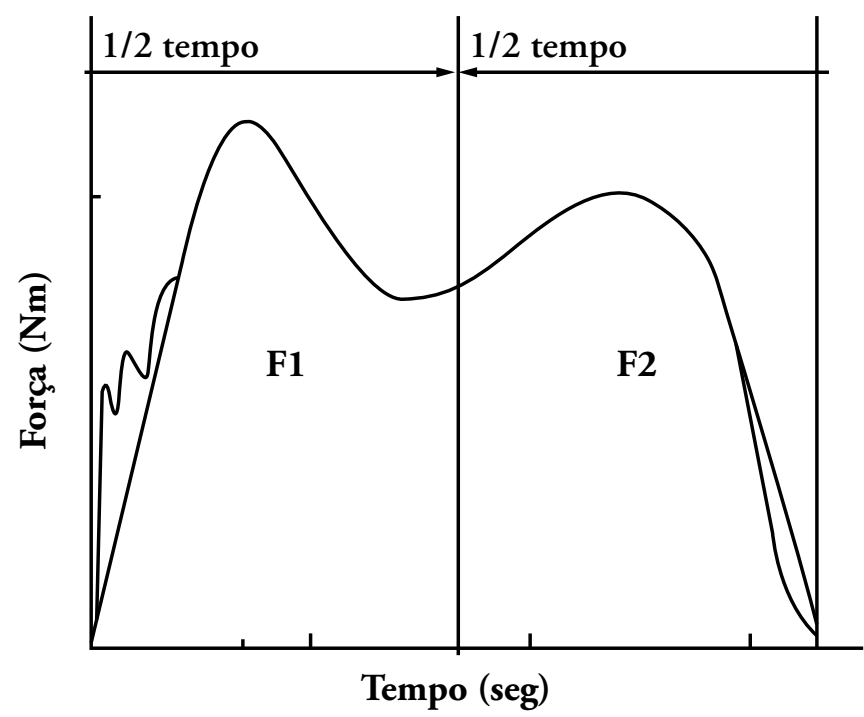

FIGURA 2 - Representação da divisão da fase de apoio da marcha para análise do pico de força de reação do solo.

TABELA 3 - Comparação das variáveis cinemáticas entre jovens, idosas com força normal e idosas com força reduzida em diferentes condições de marcha

\begin{tabular}{lccccccc}
\hline \multicolumn{8}{c}{ Variáveis cinemáticas da marcha } \\
\hline & \multicolumn{1}{c}{$\begin{array}{c}\text { Velocidade } \\
\left(\mathbf{m s} \mathbf{s}^{-1}\right)\end{array}$} & $\begin{array}{c}\text { Tempo apoio } \\
(\mathbf{s e g})\end{array}$ & $\begin{array}{c}\text { Tempo } \\
\text { balanço } \\
(\mathbf{s e g})\end{array}$ & $\begin{array}{c}\text { Tempo } \\
\text { passada } \\
(\mathbf{s e g})\end{array}$ & $\begin{array}{c}\text { Comprimento } \\
\text { do passo } \\
(\mathbf{m m})\end{array}$ & $\begin{array}{c}\text { Largura do } \\
\text { passo } \\
(\mathbf{m m})\end{array}$ \\
\hline $\begin{array}{l}\text { Condiçöes de marcha } \\
\text { Normal }\end{array}$ & GJ & $1.32 \pm 0.15$ & $0.65 \pm 0.06$ & $0.39 \pm 0.03$ & $1.04 \pm 0.08$ & $543 \pm 50$ & $175.88 \pm 26.03$ \\
& GIN & $1.22 \pm 0.16$ & $0.66 \pm 0.05$ & $0.37 \pm 0.02$ & $1.06 \pm 0.07$ & $503 \pm 45$ & $169.64 \pm 17.63$ \\
& GIR & $1.14 \pm 0.18^{*}$ & $0.69 \pm 0.06$ & $0.38 \pm 0.02$ & $1.05 \pm 0.08$ & $488 \pm 63$ & $181.41 \pm 19.14$ \\
\hline
\end{tabular}


Continuação

TABELA 3 - Comparação das variáveis cinemáticas entre jovens, idosas com força normal e idosas com força reduzida em diferentes condições de marcha

\begin{tabular}{lccccccc}
\hline \multicolumn{8}{c}{ Variáveis cinemáticas da marcha } \\
\hline & & & \multicolumn{7}{c}{$\begin{array}{c}\text { Velocidade } \\
\left(\mathbf{m s}^{-1}\right)\end{array}$} & $\begin{array}{c}\text { Tempo apoio } \\
(\mathbf{s e g})\end{array}$ & $\begin{array}{c}\text { Tempo } \\
\text { balanço } \\
(\mathbf{s e g})\end{array}$ & $\begin{array}{c}\text { Tempo } \\
\text { passada } \\
(\mathbf{s e g})\end{array}$ & $\begin{array}{c}\text { Comprimento } \\
\text { do passo } \\
(\mathbf{m m})\end{array}$ & $\begin{array}{c}\text { Largura do } \\
\text { passo } \\
(\mathbf{m m})\end{array}$ \\
\hline Carga & GJ & $1.38 \pm 0.11$ & $0.63 \pm 0.05$ & $0.38 \pm 0.02$ & $1.01 \pm 0.06$ & $537 \pm 51$ & $166.99 \pm 20.76$ \\
& GIN & $1.26 \pm 0.17$ & $0.65 \pm 0.04$ & $0.36 \pm 0.02$ & $1.04 \pm 0.06$ & $504 \pm 27$ & $170.55 \pm 18.19$ \\
& GIR & $1.16 \pm 0.16^{*}$ & $0.67 \pm 0.05$ & $0.37 \pm 0.03$ & $1.03 \pm 0.07$ & $497 \pm 50$ & $181.49 \pm 15.82$ \\
Celular & & & & & & & \\
& GJ & $1.36 \pm 0.10$ & $0.65 \pm 0.06$ & $0.36 \pm 0.03$ & $1.02 \pm 0.06$ & $550 \pm 34$ & $177.41 \pm 21.83$ \\
& GIN & $1.09 \pm 0.23^{*}$ & $0.71 \pm 0.13^{*}$ & $0.39 \pm 0.02$ & $1.11 \pm 0.05$ & $466 \pm 33^{* \dagger}$ & $181.89 \pm 26.33$ \\
& GIR & $1.02 \pm 0.25^{*}$ & $0.76 \pm 0.05^{*}$ & $0.40 \pm 0.04$ & $1.18 \pm 0.18$ & $449 \pm 58^{* \dagger}$ & $192.25 \pm 20.15$ \\
& & & & & & & \\
& GJaca & $1.40 \pm 0.12$ & $0.62 \pm 0.08$ & $0.39 \pm 0.07$ & $1.02 \pm 0.07$ & $539 \pm 56$ & $176.41 \pm 25.07$ \\
& GIN & $1.25 \pm 0.17^{*}$ & $0.67 \pm 0.05$ & $0.36 \pm 0.03$ & $1.03 \pm 0.07$ & $496 \pm 34$ & $177.30 \pm 21.89$ \\
& GIR & $1.17 \pm 0.16^{*}$ & $0.66 \pm 0.05$ & $0.37 \pm 0.03$ & $1.02 \pm 0.07$ & $488 \pm 48$ & $181.49 \pm 18.36$ \\
& & & & & & & \\
Rápida & GJ & $1.98 \pm 0.20^{\dagger}$ & $0.51 \pm 0.06^{\dagger}$ & $0.30 \pm 0.04^{\dagger}$ & $0.82 \pm 0.07^{\dagger}$ & $625 \pm 89^{\dagger}$ & $177.46 \pm 21.83$ \\
& GIN & $1.48 \pm 0.15^{* \dagger}$ & $0.58 \pm 0.04^{*^{*}}$ & $0.33 \pm 0.03^{\dagger}$ & $0.92 \pm 0.05^{* \dagger}$ & $550 \pm 46^{\dagger}$ & $177.83 \pm 11.81$ \\
& GIR & $1.54 \pm 0.15^{*}$ & $0.59 \pm 0.05^{* \dagger}$ & $0.35 \pm 0.03^{* \dagger}$ & $0.94 \pm 0.08^{* \dagger}$ & $547 \pm 50^{\dagger}$ & $186.02 \pm 24.49$ \\
\hline
\end{tabular}

\section{Análise estatística}

A análise estatística foi realizada por meio do software PASW statistics $18.0^{\circ}$ (SPSS). Para comparação dos dados cinéticos e cinemáticos entre os grupos e as condiçóes de marcha propostas foi aplicado o teste
Anova Two-Way Medidas Repetidas e o teste post-hoc de Bonferroni. A correlação entre as variáveis força reação do solo vertical, velocidade de marcha, torque extensor de joelho e idade das voluntárias foi realizada por meio do coeficiente de correlaçáo de Pearson. $\mathrm{O}$ nível de significância adotado foi de $\mathrm{p}<0.05$.

\section{Resultados}

O teste Anova Two-Way mostrou diferença estatística entre os grupos $(\mathrm{p}=0.036, \mathrm{~F}=2,134)$, condições de marcha $(\mathrm{p}<0.001, \mathrm{~F}=4.348) \mathrm{e}$ interação entre os grupos e condiçốes $(\mathrm{p}=0.001$, $\mathrm{F}=1.839)$.

Em relação à comparação entre grupos, os resultados mostraram que houve diferença significativa entre o GJ em relação aos grupos GIN e GIR para: velocidade $(\mathrm{p}=0.001, \mathrm{~F}=19.797)$, comprimento do passo $(\mathrm{p}=0.002, \mathrm{~F}=9.530)$, tempo de apoio $(\mathrm{p}=0.045, \mathrm{~F}=20.016)$, PFRSV na aceitação do peso $(\mathrm{p}=0.005, \mathrm{~F}=7.453)$, PFRSV na propulsão $(\mathrm{p}<0.001, \mathrm{~F}=19.467)$, taxa de aceitação do peso $(\mathrm{p}=0.012, \mathrm{~F}=6.600)$ e taxa de propulsão $(\mathrm{p}=0.001, \mathrm{~F}=14,824)$. Não houve diferença significativa entre GIR e GIN para as variáveis analisadas.

Para análise da interação entre os grupos, os resultados mostraram que durante a condiçáo de marcha I as variáveis PFRSV na fase de propulsão e comprimento do passo foram, respectivamente, $9 \%$ e $11 \%$ inferior para o GIR ( $\mathrm{p}=0.005$, $\mathrm{p}=0,035)$ em relação às voluntárias jovens. Durante a condiçáo de marcha falando ao celular observamos que GIN e GIR apresentaram, respectivamente, redução da velocidade de marcha $(\mathrm{p}=0.004$, $\mathrm{p}=0.006)$, comprimento do passo $(\mathrm{p}<0.001$, $\mathrm{p}<0.001)$, tempo de apoio $(\mathrm{p}=0.038, \mathrm{p}=0.035)$, 
PSRSV na propulsão $(\mathrm{p}<0.001, \mathrm{p}=0.002)$ e taxa de propulsão $(\mathrm{p}=0.035, \mathrm{p}=0.001)$ em relação as voluntárias jovens, assim como durante a condição de marcha com dupla tarefa motora (IV) para as variáveis velocidade de marcha $(\mathrm{p}=0.016, \mathrm{p}=0.015)$, tempo de apoio $(\mathrm{p}=0.016, \mathrm{p}=0.015)$ e PFRSV na fase de propulsão $(\mathrm{p}=0.006, \mathrm{p}=0.020)$.

Para a condição de marcha em velocidade rápida os grupos GIN e GIR apresentaram valores inferiores as voluntárias jovens para as variáveis velocidade ( $\mathrm{p}<0.001, \mathrm{p}<0.001)$, comprimento do passo $(\mathrm{p}=0.012, \mathrm{p}=0.019)$, tempo de apoio $(\mathrm{p}=0.001$, $\mathrm{p}=0.043)$, tempo de passada $(\mathrm{p}=0.012, \mathrm{p}=0.03)$, PFRSV nas fases de propulsão $(\mathrm{p}=0.004, \mathrm{p}=0.029)$ e aceitaçáo do peso $(\mathrm{p}=0.044, \mathrm{p}=0.001)$, taxa de propulsão $(\mathrm{p}<0.001, \mathrm{p}=0.002)$ e taxa de aceitação do peso $(\mathrm{p}=0.046, \mathrm{p}=0.014)$.

A análise intergrupo mostrou que durante a condiçáo de marcha rápida o GJ e GIN apresentaram aumento da taxa de aceitação do peso/propulsão e PFRSV na fase de aceitação do peso ( $\mathrm{p}<0.001$ para todos) em relação a condição de marcha normal. Em relação as varáveis cinemáticas, a análise intergrupo mostrou que os três grupos analisados apresentaram diferenças significativas para a condição de marcha rápida em relação a marcha normal para todas as variáveis analisadas $(\mathrm{p}<0.05)$. Além disso, o GIN apresentou redução de $8 \%$ no comprimento do passo durante a condição de marcha III em relação a condição I.

O GIR apresentou diferença entre a condição de marcha normal em relação à marcha carregando sacolas para as variáveis PFRSV na fase de aceitação do peso e propulsão $(\mathrm{p}=0.008$, $\mathrm{p}=0.002$, respectivamente) e na taxa de propulsão $(p=0.040)$; e também em relação à condição de marcha falando ao celular para a variável comprimento do passo $(\mathrm{p}=0.001)$.

A TABELA 4 apresenta a correlação entre a força reaçáo do solo vertical, velocidade de marcha, torque extensor de joelho e idade das voluntárias.

TABELA 4 - Correlação entre força reação do solo, velocidade de marcha, torque extensor de joelho e idade das voluntárias

\begin{tabular}{lccc}
\hline & $\begin{array}{c}\text { PFRSV aceitaçáo } \\
\text { do peso }\end{array}$ & PFRSV propulsão & Torque Extensor de Joelho \\
\hline Idade & -0.285 & $-0.455^{* *}$ & $-0.652^{* *}$ \\
Velocidade de preferência & 0.192 & $0.204^{*}$ & $0.344^{*}$ \\
Velocidade de preferência máxima & 0.247 & 0.325 & $0.561^{* *}$ \\
PFRSV aceitação do peso & - & $0.411^{* *}$ & 0.279 \\
PFRSV propulsão & $0.411^{* *}$ & - & $0.513^{* *}$ \\
\hline
\end{tabular}

*Correlação significativa para p< 0.05; **Correlação significativa para $p<0.01$.

\section{Discussão}

Esse estudo procurou investigar o comportamento de variáveis cinéticas e cinemáticas da marcha em indivíduos idosos com força muscular normal e reduzida durante condiçóes de marcha que fazem parte do cotidiano. Apesar de ambos os grupos de idosos terem apresentado alteraçôes no padrão de marcha em relação às voluntárias jovens, essas foram mais evidenciadas em idosas com força muscular reduzida.

\section{Efeito da diminuição da força muscular nas variáveis da marcha}

Alteraçóes biomecânicas durante a marcha simples em idosas são mostradas na literatura e caracterizam- se por um padrão de marcha instável e irregular que predispóe esses indivíduos a maior risco de quedas, assim como no presente estudo, as voluntárias que apresentaram maior comprometimento das variáveis cinéticas e cinemáticas da marcha também relataram maior número de quedas nos últimos doze meses. Os resultados desse estudo mostraram que as variáveis comprimento de passo e PFRSV na fase de propulsão diferenciou idosos com baixa força muscular de indivíduos jovens durante a marcha em velocidade de preferência. Esse achado corrobora os estudos de BAIRD et al. ${ }^{24}$ e LARoche et al..$^{20}$, na qual as idosas com déficits de força apresentaram alteraçóes de parâmetros espaciais e temporais da marcha, sugerindo que essas mudanças biomecânicas podem ser consideradas 
como estratégia compensatória para manutenção do equilíbrio e do estado metabólico a fim de evitar fadiga, sendo que a consequência dessas alteraçôes seria a redução da velocidade de marcha que pode contribuir para déficits ainda maiores de mobilidade ${ }^{13,20}$.

As idosas com baixa força muscular desse estudo caminharam com valores de velocidade de preferência abaixo de $1.22 \mathrm{~m} \mathrm{~s}^{-1}$, critério frequentemente utilizado para indicar limitaçôes de mobilidade ${ }^{23}$. A redução da força muscular com o avanço da idade é um dos principais fatores associados a diminuiçáo da velocidade de marcha em idosos ${ }^{24}$. O declínio progressivo dos níveis de força acompanhado da redução da velocidade de marcha pode comprometer a mobilidade até que as idosas náo tenham força suficiente para caminhar em velocidades funcionalmente significativas ${ }^{13,22}$. Os resultados desse estudo mostraram que as variáveis velocidade de preferência e velocidade de preferência máxima foram correlacionadas com o torque extensor de joelho das voluntárias. Essa correlação demonstra a menor capacidade de idosas com força muscular reduzida aumentarem sua velocidade de marcha quando solicitado e consiste com os achados do estudo de Kwon et al. ${ }^{25}$, no qual foi observado que os acréscimos na velocidade de marcha ocorrem de maneira linear ao aumento da força dos extensores de joelho, indicando que o quadríceps é um dos principais grupos musculares responsáveis pelo aumento da velocidade de marcha ${ }^{23,24}$.

Estudos prévios têm mostrado que a velocidade de caminhada e comprimento do passo apresentam uma relação linear com o componente vertical da força de reação do solo ${ }^{26}$. Nossos resultados mostraram que as voluntárias idosas de ambos os grupos apresentaram menores valores de velocidade e consequentemente de picos e taxas de força de reação do solo na fase de aceitação do peso e propulsão, sendo essas alteraçôes mais evidenciadas em idosas com força muscular reduzida. Esses achados são consistentes com aqueles apresentados por STACOFF et al..$^{23}$, que encontraram diferença nos valores de pico e taxa de força de reação durante a marcha entre jovens e idosos, atribuindo essas diferenças ao efeito da idade na velocidade de marcha e consequentemente nas variáveis cinéticas ${ }^{20}$. YAMAda e Demura ${ }^{27}$ encontraram resultados semelhantes durante a tarefa de sentar e levantar da cadeira em indivíduos idosos, inferindo que a redução dos parâmetros de força de reação do solo estão associados a menor capacidade física e maior risco de quedas nessa população ${ }^{28}$.
As idosas com déficit de força desse estudo obtiveram valores de taxa de propulsão inferior em relação a jovens para todas as condições de marcha sendo estatisticamente diferente em condições de dupla tarefa e marcha rápida, o que pode ter comprometido o desempenho das voluntárias durante essas condiçóes de marcha. Esses achados são consistentes com LARoche et al. ${ }^{20}$, que encontraram relação positiva entre a força dos membros inferiores e a taxa de propulsão, indicando que a reduçáo da taxa de propulsão em idosos pode afetar negativamente a mobilidade ${ }^{13}$. Segundo Winter ${ }^{29}$ a fase de propulsão da marcha é fundamental para garantir a eficiência do próximo passo $^{28}$. A propulsão corresponde à segunda metade da fase de apoio da marcha, na qual os músculos flexores plantares geram maior ativação para simultaneamente manter o suporte do peso corporal e realizar a progressão anterior do tronco e perna ${ }^{29,30}$. $\mathrm{O}$ aumento do trabalho exercido pelos flexores plantares resulta em acúmulo de energia elástica no tendão que irá contribuir para aumento da taxa de propulsão ocasionando maior aceleração do corpo no sentido vertical e horizontal anterior ${ }^{29,30}$. Estudos prévios têm demonstrado redução significativa da força muscular com a idade em membros inferiores, sendo mais acentuada nos flexores plantares ${ }^{31,32}$, o que pode justificar o comprometimento da função desses músculos em impulsionar o corpo para frente resultando em menor taxa de propulsão e consequentemente comprimento de passo e velocidade de marcha.

Além disso, nesse estudo a variável torque extensor de joelho apresentou correlação positiva com a velocidade e PFRSV na fase de propulsão, indicando que maiores déficits de força em idosos estão associados a menores valores de velocidades de marcha e força de reação na fase de propulsão, que pode comprometer o desempenho desses indivíduos durante a marcha. De acordo com Plummer-D’Amato et al. ${ }^{33}$, essas alteraçóes podem representar o impacto do envelhecimento na biomecânica da marcha, que náo se limita a redução da função motora mas uma mudança no controle da marcha, na qual os grupos musculares de membros inferiores, principalmente do tornozelo, aumentam o percentual de cocontração como estratégia compensatória para diminuição do equilíbrio dinâmico e isso pode levar a menor capacidade de gerar força na fase de propulsão e consequentemente uma velocidade de marcha mais lenta em idosos ${ }^{34}$. 


\section{Efeito da dupla tarefa nas variáveis de marcha}

Esse estudo propôs avaliar condições de marcha que simulassem atividades que fazem parte do cotidiano da populaçáo idosa e observamos que a dupla tarefa afeta significativamente alguns parâmetros cinéticos e cinemáticos da marcha. Ou seja, a realização de tarefas simultâneas afeta o padrão de marcha, o que confirma que esse movimento não é automático, mas precisa de um controle cognitivo.

Durante a condição de marcha falando ao celular, verificou-se que as idosas apresentaram reduçáo do comprimento do passo, tempo de apoio, velocidade de marcha e do PFRSV e da taxa na fase de propulsão em relação a jovens. Além disso, o GIR apresentou valores inferiores de taxa de aceitação do peso e propulsão em relação ao GIN. Esses resultados estáo de acordo com Nordin et al. ${ }^{35}$, que encontraram menor comprimento e largura do passo ao solicitar que idosos caminhassem enumerando verbalmente diferentes tipos de animais, sugerindo que as alteraçóes cinemáticas proporcionadas pela dupla tarefa cognitiva refletem uma falha do sistema nervoso central para planejar tarefas simultaneamente e estão associadas a maior risco de quedas nesses indivíduos ${ }^{36}$. Plummer-D'Amato et al. ${ }^{33}$ encontraram diminuição da velocidade durante a marcha com narrativa espontânea de $10 \%$ em jovens e $20 \%$ em idosos, sugerindo que o maior comprometimento da velocidade em idosos se deve a distração causada por uma tarefa cognitiva que afeta em proporçôes menores outras populaçôes ${ }^{37}$. De acordo com Nordin et al..$^{35}$ e Mersmann et al. ${ }^{38}$, o declínio da capacidade de processamento de informaçóes com o avanço da idade faz com que a condição de dupla tarefa afete a marcha, a segunda atividade ou ambas dependendo da prioridade e complexidade da tarefa ${ }^{32,33}$. Situaçóes de alta demanda atencional, como por exemplo falar ao telefone, são priorizadas pelo sistema nervoso central fazendo com que a performance da marcha seja prejudicada e isso pode resultar em alteraçóes cinemáticas e cinéticas que predispóe idosos a maior risco de quedas ${ }^{2,32,33,39}$, assim como observado no presente estudo.

Para a marcha com obstrução da visão dos pés (IV) foi observado diferença significativa para as variáveis velocidade de marcha e PFRSV e taxa na fase de propulsão entre jovens e idosos com baixa força muscular. RietDyK e RHEA ${ }^{40}$ demonstraram que a informação visual em idosos é importante para determinar a trajetória de movimento dos membros inferiores, diminuindo a probabilidade de ocorrer tropeços ${ }^{41}$. Bock e BeURsKens ${ }^{42}$ encontram diferença para o custo da tarefa em jovens e idosos ao comparar a marcha simples com a marcha carregando uma placa opaca, sendo que essa diferença foi maior para os idosos ressaltando a importância da visão dos pés para eles executarem um padrão de marcha estável, garantindo o posicionamento correto do pé no solo ${ }^{39}$. Essa condição de marcha pode ter proporcionado um desafio maior para voluntárias idosas, principalmente para aquelas com déficits de força pois apresentaram menor PFRSV na fase de propulsão em relação as idosas com força normal, que resultou na redução da velocidade como estratégia para conseguir executar a tarefa com segurança.

Durante a caminhada carregando cargas correspondente a $10 \%$ do peso corporal, as voluntárias idosas com força muscular reduzida apresentaram aumento do PFRSV na fase aceitação do peso e na taxa de propulsão em relação à marcha simples e inferior aos valores apresentados pelas jovens. Nos estudos de Browning e Kram ${ }^{43}$ e LaRoche et al. ${ }^{44}$ indivíduos idosos obesos apresentaram maiores picos e taxas de força de reação em relação aos idosos sem obesidade, demonstrando que as maiores forças aplicadas entre o pé e o solo resultante do aumento da massa corporal são refletidas nos picos de força de reação do solo ${ }^{44,45}$. HoF $^{46}$ assume que a força de reação é proporcional a força produzida para suportar o peso corporal ${ }^{47}$. No presente estudo as voluntárias podem ter aumentado a proporção das forças imposta às articulaçóes do membro inferior a fim de produzir força suficiente para suportar o peso corporal somado as respectivas cargas que elas carregavam, resultando no aumento dos valores da força de reação do solo durante a aceitação do peso. De acordo com Qu e YeO ${ }^{48}$ e ATtwells et al. ${ }^{49}$, essas alteraçóes podem ser consideradas uma estratégia de proteção para auxiliar na absorção das forças de impacto que são maiores durante o transporte de carga ${ }^{50,51}$.

Este estudo encontrou que apenas as condiçóes de marcha com dupla tarefa foram capazes de diferenciar idosos com força normal daqueles com força reduzida, em relaçáo as variáveis cinéticas da marcha. Podemos considerar a dupla tarefa como um padrão de avaliação superior para identificar idosos com comprometimento biomecânico da marcha. 
Efeito do aumento da velocidade nas variáveis da marcha

A marcha em velocidade de preferência máxima proporcionou significativas alteraçóes nas variáveis cinéticas e cinemáticas nos três grupos avaliados em relaçáo àquelas apresentadas durante as demais condiçóes de marcha.

Em relação as variáveis cinéticas, os grupos GJ e GIN apresentaram aumento do PFRSV na aceitação do peso e nas taxas de força de reação do solo durante a marcha rápida em relação a marcha em velocidade de preferência. Esses achados são consistentes com aqueles apresentados por Chung e WAng ${ }^{52}$, que observaram o efeito da velocidade na força de reação do solo, sendo que o aumento do pico de força de reaçáo com o acréscimo da velocidade representa a maior demanda imposta aos músculos dos membros inferiores para conter excentricamente a força externa ${ }^{44}$. O GIR por sua vez, não apresentou diferença significativa para essas variáveis e isso pode estar relacionado com diminuição da reserva muscular fisiológica nesse grupo, que faz com que esses indivíduos realizem suas atividades diárias muito próximas de suas capacidades máximas, ou seja, eles não apresentam força muscular suficiente para aumentar a velocidade de marcha quando solicitado ${ }^{46}$.

Em relação às variáveis cinemáticas, o aumento da velocidade em diferentes proporçóes em jovens, idosas com mobilidade normal e mobilidade reduzida, sendo 50\%, 29\% e 26\% em relação à velocidade de preferência, respectivamente, pode ser interpretado como uma estratégia mais cautelosa para as idosas conseguirem conter as maiores forças externas ocasionadas por velocidades mais rápidas e assim manter um melhor controle do equilíbrio durante a marcha. A variável comprimento do passo apresentou aumento significativo durante a marcha rápida para os três grupos analisados, sendo essa diferença em menores proporçóes para idosas com mobilidade reduzida.

Os resultados desse estudo corroboram com os estudos de LARSEN et al..$^{53}$ e LARoche et al. ${ }^{44}$ que observaram alteraçóes dos parâmetros cinéticos e cinemáticos durante a transição na escada e na marcha, respectivamente, ao comparar velocidade habitual para execução da tarefa com a velocidade máxima, indicando que as idosas apresentaram menor capacidade de reserva muscular para aumentar o comprimento do passo e a velocidade quando solicitado $^{13,47}$. A diminuição da força muscular com o envelhecimento faz com que as idosas executem a marcha próximo de suas capacidades máximas, que pode resultar em fadiga muscular e levar ao comprometimento da habilidade de se movimentar rapidamente que se torna fundamental para se recuperar após perturbação do equilíbrio ou para atravessar a rua com segurança, por exemplo ${ }^{13,47}$.

A diminuição do torque extensor de joelho contribui para menor velocidade de marcha e força de reaçáo do solo e quando associado às alteraçóes cinemáticas da marcha pode comprometer a execução de um padrão de marcha eficiente tanto em condiçóes simples como durante dupla-tarefa, e resultar em menor independência, maior risco de incapacidade funcional e quedas nessa população. Esses resultados podem oferecer subsídios para que os programas de exercícios físicos para idosos abordem atividades voltadas para o fortalecimento dos membros inferiores, principalmente dos extensores de joelho e flexores plantares.

\section{Abstract}

Influence of low strength in elderly in the biomechanical parameters of the gait

The aim of the study was to analyze how lower limb strength of knee extensors affects biomechanical variables during simple gait and dual task. Study participants were 15 young (GJ), 14 older adult women with normal strength (GIN) and 13 elderly women with low strength (GIR). The strength level was determined by the knee extensor torque, considering the threshold of $1.5 \mathrm{NKg}-1$. Biomechanical gait assessment was made using a 3D analysis with a force plate. The conditions were analyzed in habitual gait (I), walking while carrying a load (II), walking while talk on the mobile phone (III), walking while carrying a plate (IV) and fast gait (V). The results showed that GIR and GIN showed kinetic and kinematic changes during gait conditions with dual motor task and cognitive task in relation to GJ. However only the variables propulsion rate and the vertical ground reaction force $(p<0.05)$ during the situation with dual task were able to differentiate the elderly groups. Thus, we can conclude that the assessment of gait with dual task is able to differentiate 
elderly with muscle strength impairment. In addition, the strength of the knee extensors is determining the gait speed parameters and ground reaction force, so the weakness of that muscle group associated with kinematic changes of gait may compromise the execution of a pattern of efficient and safe, during simple gait and dual-task, resulting in increased risk of falls and functional disability.

KEYWORDS: Dual-task; Ground Reaction Force; Kinematics.

\section{Conflito de interesses}

Os autores declaram que não há conflito de interesses associado a este estudo.

\section{Agradecimentos}

À Fundação de Amparo à Pesquisa do Estado de São Paulo (Fapesp) e ao Conselho Nacional de Desenvolvimento Científico e Tecnológico (CNPq).

\section{Referências}

1. Callisaya ML, Blizzard L, Schmidt MD, et al. Gait, gait variability and the risk of multiple incident falls in older people: a population-based study. Age and Ageing. 2011;40(4):481-7.

2. Hollman JH, Childs KB, Mueller AC, McNeil ML, Quilter CM, Youdas JW. Number of strides required for reliable measurement of pace, rhythm and variability parameters of gait during normal and dual task walking in older individuals. Gait Posture 2010;32(1):23-8.

3. Jahn K, Zwergal A, Schniepp R. Gait disturbances in old age. Dtsch Arztebl Int. 2010;107(17):306-16.

4. Verhoeff LL, Horlings CG, Janssen LJ, Bridenbaugh SA, Allum JH. Effects of biofeedback on trunk sway during dual tasking in the healthy young and elderly. Gait Posture. 2009;30(1):76-81.

5. Bock O. Dual-task costs while walking increase in old age for some, but not for other tasks: an experimental study of healthy young and elderly persons. J Neuroeng Rehabil. 2011;5:27.

6. Muhaidat J, Kerr A, Evans JJ, Pilling M, Skelton DA. Validity of simple gait-related dual-task tests in predicting falls in community-dwelling older adults. Arch Phys Med Rehabil. 2014;95(1):58-64.

7. Hallal CZ, Marques NR, Castro A, et al. Variabilidade de parâmetros eletromiográficos e cinemáticos em diferentes condiçóes de marcha de idosos. Motriz. 2013;19(1):141-50.

8. Hallal CZ, Marques NR, Spinoso DH, Vieira ER, Gonçalves M. Electromyographic patterns of lower limb muscles during apprehensive gait in younger and older female adults. J Electromyogr Kinesiol. 2013;23(5):1145-9.

9. Lockhart TE, Woldstad JC, Smith JL. Effects of age-related gait changes on the biomechanics of slips and falls. Ergonomics. 2003;46(12):1136-60.

10. Bean JF, Leveille SG, Kiely DK, Bandinelli S, Guralnik JM, Ferruci L. A comparison of leg power and leg strength within the In CHIANTI study: which influences mobility more? J Gerontol A Biol Sci Med Sci. 2003;58(8):728-33.

11. Hicks GE, Shardell M, Alley DE, et al. Absolute strength and loss of strength as predictors of mobility decline in older adults: the In CHIANTI study. J Gerontol A Biol Sci Med Sci. 2012;67(1):66-73.

12. Mänty M, de Leon CF, Rantanen T, et al. Mobility-related fatigue, walking speed, and muscle strength in older people. J Gerontol A Biol Sci Med Sci. 2012;67(5):523-9.

13. Ploutz-Snyder LL, Manini T, Ploutz-Snyder RJ, Wolf DA. Functionally relevant thresholds of quadriceps femoris strength. J Gerontol A Biol Sci Med Sci. 2002;57(4):144-52.

14. Manini TM, Visser M, Won-Park S, et al. Knee extension strength cutpoints for maintaining mobility. J Am Geriatr Soc. 2007;55(3):451-7. 
15. Marko M, Neville CG, Prince MA, Ploutz-Snyder LL. Lower-extremity force decrements identify early mobility decline among community-dwelling older adults. Phys Ther. 2012;92(9):1148-59.

16. Rosso AL, Auchincloss AH, Michael YL. The urban built environment and mobility in older adults: a comprehensive review. J Aging Res. 2011;816106.

17. de Vries NM, van Ravensberg CD, Hobbelen JS, Olde Rikkert MG, Staal JB, Nijhuis-van der Sanden MW. Effects of physical exercise therapy on mobility, physical functioning, physical activity and quality of life in community-dwelling older adults with impaired mobility, physical disability and/or multi-morbidity: a meta-analysis. Ageing Res Rev. 2012;11(1):136-49.

18. Knaggs JD, Larkin KA, Manini TM. Metabolic cost of daily activities and effect of mobility impairment in older adults. J Am Geriatr Soc. 2011;59(11):2118-23.

19. Hartmann A, Knols R, Murer K, de Bruin ED. Reproducibility of an isokinetic strength-testing protocol of the knee and ankle in older adults. Gerontology. 2009;55(3):259-68.

20. LaRoche DP, Millett ED, Kralian RJ. Low strength is related to diminished ground reaction forces and walking performance in older women. Gait Posture. 2011;33(4):668-72.

21. Shiavi R, Frigo C, Pedotti A. Electromyographic signals during gait: criteria for envelope filtering and number of strides. Med Biol Eng Comput. 1998;36(2):171-8.

22. Hamill J, Knutzen KM, Derrick TR. Bases biomecânicas do movimento humano. 3a ed. Barueri: Manole; 2012.

23. Stacoff A, Diezi C, Luder G, Stüssi E, Quervain IAK. Ground reaction forces on stairs: effects of stair inclination and age. Gait Posture. 2005;21(1):24-38.

24. Baird JL, Van Emmerick REA. Young and older adults use different strategies to perform a standing turning task. Clin Biomech. 2009;24(10):826-32.

25. Kwon IS, Oldaker S, Schrager M, Talbot LA, Fozard JL, Metter EJ. Relationship between muscle strength and the time taken to complete a standardized walk-turn-walk test. J. Gerontol A Biol Sci Med Sci. 2001;56(9):B398-404.

26. Rantanen T, Avela J. Leg extension power and walking speed in very old people living independently. J Gerontol A Biol Sci Med Sci. 1997;52(4):225-31.

27. Yamada T, Demura S. Relationships between ground reaction force parameters during a sit-to-stand movement and physical activity and falling risk of the elderly and a comparison of the movement characteristics between the young and the elderly. Arch Gerontol Geriatr. 2009;48(1):73-7.

28. Khokhar SR, Stern Y, Bell K, et al. Persistent mobility deficit in the absence of deficits in activities of daily living: a risk factor of mortality. J Am Geriatr Soc. 2001;49(11):1536-43.

29. Winter DA. Overall principle of lower limb support during stance phase of gait. J Biomech. 1980;13(11):923-7.

30. Harries UJ, Bassey EJ. Torque-velocity relationships for the knee extensors in women in their 3rd and 7th decades. Eur J Appl Physiol Occup Physiol. 1990;60(3):187-90.

31. Keller TS, Weisberger AM, Ray JL, Hasan SS, Shiavi RG, Spengler DM. Relationship between vertical ground reaction force and speed during walking, slow jogging, and running. Clin Biomech. 1996;11(5):253-9.

32. Liu MQ, Anderson FC, Pandy MG, Delp SL. Muscles that support the body also modulate forward progression during walking. J Biomech. 2006;39(14):2623-30.

33. Plummer-D’Amato P, Altmann LJ, Reilly K. Dual-task effects of spontaneous speech and executive function on gait in aging: exaggerated effects in slow walkers. Gait Posture. 2011;33(2):233-7.

34. Farley CT, Ferris DP. Biomechanics of walking and running: from center of mass movement to muscle action. Exerc Sport Sci Rev. 1998;26:253-85.

35. Nordin E, Moe-Nilssen R, Ramnemark A, Lundin-Olsson L. Changes in step-width during dual-task walking predicts falls. Gait Posture. 2010;32(1):92-7.

36. Reeves ND, Spanjaard M, Mohagheghi AA, Baltzopoulos V, Maganaris CN. Older adults employ alternative strategies to operate within their maximum capabilities when ascending stairs. J Electromyogr Kinesiol. 2009;19(2):57-68.

37. Marques NR, Hallal CZ, Crozara LF, et al. Lower limb strength is associated with gait biomechanical abnormalities in older female fallers and non-fallers. Isokinet Exerc Sci. 2013; 21(2):151-9.

38. Mersmann F, Bohm S, Bierbaum S, Dietrich R, Arampatzis A. Young and old adults prioritize dynamic stability control following gait perturbations when performing a concurrent cognitive task. Gait Posture. 2013;37(3):373-7.

39. Thelen DG, Schultz AB, Alexander NB, Ashton-Miller JA. Effects of age on rapid ankle torque development. J Gerontol A Biol Sci Med Sci. 1996;51(5):226-32.

40. Rietdyk S, Rhea CK. Control of adaptive locomotion: effect of visual obstruction and visual cues in the environment. Exp Brain Res. 2006;169(2):272-8. 
41. Taylor ME, Delbaere K, Mikolaizak AS, Lord SR, Close JT. Gait parameter risk factors for falls under simple and dual task conditions in cognitively impaired older people. Gait Posture. 2013;37(1):126-30.

42. Bock O, Beurskens R. Age-related deficits of dual-task walking: the role of foot vision. Gait Posture. 2011;33(2):190-4.

43. Browning RC, Kram R. Effects of obesity on the biomechanics of walking at different speeds. Med Sci Sports Exerc. 2007;39(9):1632-41.

44. LaRoche DP, Kralian RJ, Millett ED. Fat mass limits lower-extremity relative strength and maximal walking performance in older women. J Electromyogr Kinesiol. 2011;21(5):754-61.

45. Raz N. Aging of the brain and its impact on cognitive performance: integration of structural and functional findings. In: Craik FIM, Salthouse TA, editors. The handbook of aging and cognition. Mahwah: Lawrence Erlbaum; 2000. p. 1-90.

46. Hof AL. On the interpretation of the support moment. Gait Posture. 2000;12(3):196-9.

47. Chapman GJ, Hollands MA. Evidence for a link between changes to gaze behaviour and risk of falling in older adults during adaptive locomotion. Gait Posture. 2006;24(3):288-94.

48. Qu X, Yeo JC. Effects of load carriage and fatigue on gait characteristics. J Biomech. 2011;44(7):1259-63.

49. Attwells RL, Birrell SA, Hooper RH, Mansfield NJ. Influence of carrying heavy loads on soldiers' posture, movements and gait. Ergonomics. 2006;49(14):1527-37.

50. Marigold DS. Negotiating varying ground terrain during locomotion: insights into the role of vision and the effects of aging [tese]. Waterloo (ON): University of Waterloo; 2006.

51. Crosbie J, Vachalathiti R, Smith R. Patterns of spinal motion during walking. Gait Posture. 1997;5(1):6-12.

52. Chung MJ, Wang MJ. The change of gait parameters during walking at different percentage of preferred walking speed for healthy adults aged 20-60 years. Gait Posture. 2010;31(1):131-5.

53. Larsen AH, Sørensen H, Puggaard L, Aagaard P. Biomechanical determinants of maximal stair climbing capacity in healthy elderly women. Scand J Med Sci Sports. 2009;19(5):678-86.

ENDEREÇO

Deborah Hebling Spinoso Av. Higino Muzzi Filho, 737 17525-900 - Marília - BRASIL e-mail: deborahebling@yahoo.com.br
Recebido para publicação: 29/07/2016

1a. Revisão: 15/11/2016

2a. Revisão: 03/03/2017

Aceito: 15/03/2017 